\title{
Alliance Formation of Unmanned Aerial Vehicle based on Holon
}

\author{
Bowen Han a, Peiyang Yao ${ }^{b}$ \\ School of Air Force Engineering University, Xian 710077, China \\ ahwyyxlw@163.com, b18066619922@163.com
}

Keywords: Alliance, Hybrid Bee Colony Algorithm, Multi-Objective Optimization.

\begin{abstract}
The assignment problem of the unmanned aerial vehicle (UAV) cluster to target group is analyzed based on the Holon theory. The formation process of the air fleet Holon alliance is the process of the unordered UAV cluster form into columns satisfying the tasks demand. The total task of the target group is decomposed into different types of sub tasks, defining the cluster resource redundancy index and the resource redundancy variance index according to the UAV resource capability and task resource requirement, a multi-objective optimization model of alliance formation is established.
\end{abstract}

\section{Introduction}

The successful application of unmanned combat system makes the future cluster operations become a trend. There are successful applications to modern combat operations from the land combat robot to the sea unmanned surface craft, and then to the air UAV. As an unmanned combat platform, UAV has the advantages of zero casualties, high mobility and low cost. However, due to the limitation of single UAV combat capability, single UAV operational mode is developing towards unmanned aerial combat mode [1].

Taking the operation of cluster as background, using Holon theory, this paper builds a model of Holon alliance of unmanned vehicle swarm (HAVS) formation, and presents a multi-objective mixed colony algorithm to solve the model. The rationality of model and effectiveness of the algorithm are verified.

\section{Mathematical description of HAVS}

In actual combat, in the face of the operation situation of large target group, all the tasks in each target group are the alliance tasks of the formation alliance, and all alliances complete the combat task. Therefore, the effect of the UAV group on the target group depends on the formation of each UAV alliance for each target group.

\subsection{HAVS task decomposition}

The total combat mission of a cluster to target group can be decomposed into

$T=\left\{\left(t_{1}^{D}, t_{1}^{A}, t_{1}^{E}\right),\left(t_{2}^{D}, t_{2}^{A}, t_{2}^{E}\right), \cdots,\left(t_{N}^{D}, t_{N}^{A}, t_{N}^{E}\right)\right\}$

Where $t_{i}^{D}$ represents reconnaissance mission, $t_{i}^{A}$ represents attack mission, $t_{i}^{E}$ represents evaluate mission.

\subsection{Design of HAVS formation}

On the basis of task decomposition, HAVS can be described as follows.

Each formation alliance $A_{k} \in H$ is regarded as a "virtual UAV" with the ability vector $B_{A_{k}}=\left[b_{A_{k}}^{D}, b_{A_{k}}^{A}, b_{A_{k}}^{E}\right]$, which $b_{A_{k}}^{D}, b_{A_{k}}^{A}, b_{A_{k}}^{E}$ is the total capability of all UAV Holon.

The task capability requirements for the alliance task $T=\left\{\left(t_{1}^{D}, t_{1}^{A}, t_{1}^{E}\right),\left(t_{2}^{D}, t_{2}^{A}, t_{2}^{E}\right), \cdots,\left(t_{N}^{D}, t_{N}^{A}, t_{N}^{E}\right)\right\}$ can be described as a vector $D_{T_{k}}=\left[d_{T_{k}}^{D}, d_{T_{k}}^{A}, d_{T_{k}}^{E}\right]$.

HAVS formation is to form $N_{A}$ Holon alliance corresponding to the task group $T_{1}, T_{2}, \cdots, T_{N_{A}}$ to minimize the average resource capacity redundancy under the premise of meeting the task requirements. 


\section{Mathematical model of HAVS formation}

This section first gives the measure of HAVS resource redundancy, and then establishes multi-objective optimization model of HAVS formation problem.

\subsection{Resource redundancy measure}

The resource redundancy of a single alliance is defined as

$\eta_{k}=w_{i}^{D}\left(b_{A_{k}}^{D}-d_{T_{k}}^{D}\right)+w_{i}^{A}\left(b_{A_{k}}^{A}-d_{T_{k}}^{A}\right)+w_{i}^{E}\left(b_{A_{k}}^{E}-d_{T_{k}}^{E}\right)$

Where $w_{i}^{D}, w_{i}^{A}, w_{i}^{E}$ are resource redundancy coefficients of reconnaissance, attack and evaluate.

\subsection{Multi-objective optimization model}

We can see that the more efficient the cluster resource allocation is, the more effective the overall operational effectiveness is, so the objective function can be described as following:

(1) Minimizing the average resource capacity redundancy $\eta$ of HAVS

$$
\min \eta=\frac{1}{N_{A}} \sum_{k=1}^{N_{A}} \eta_{k}
$$

(2) Minimize the variance $D_{\eta}$ of resource capacity redundancy of HAVS

$$
D_{\eta}=\frac{1}{N_{A}-1} \sum_{k=1}^{N_{A}}\left[w_{i}^{D}\left(b_{A_{k}}^{D}-d_{i}^{D}\right)+w_{i}^{A}\left(b_{A_{k}}^{A}-d_{i}^{A}\right)+w_{i}^{E}\left(b_{A_{k}}^{E}-d_{i}^{E}\right)-\eta\right]^{2}
$$

The overall resource capability of a single formation alliance must satisfy the resource capacity requirements of the alliance task.

$$
b_{A_{k}}^{j} \geq d_{T_{k}}^{j}, \forall b_{A_{k}}^{j} \in B_{A_{k}}, d_{T_{k}}^{j} \in D_{T_{k}}
$$

In summary, the multi-objective optimization model of HAVS formation can be described as following:

$$
\begin{gathered}
\min f=\left(\eta, D_{\eta}\right) \\
b_{A_{k}}^{j} \geq d_{T_{k}}^{j}, \forall b_{A_{k}}^{j} \in B_{A_{k}}, d_{T_{k}}^{j} \in D_{T_{k}}
\end{gathered}
$$

\section{Model solving}

Artificial bee colony algorithm is a new algorithm proposed in 2005[2], which has great superiority in solving the optimization problem. The hybrid bee colony algorithm is proposed in literature [3] to improve the search performance and precision. In this paper, a multi-objective hybrid bee colony algorithm (MHBC) is proposed to solve the optimization model (6).

\subsection{Algorithm flow}

Specific flow of MHBC algorithm is shown in figure1.

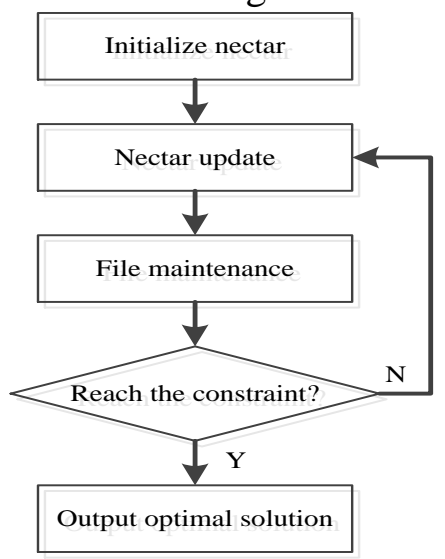

Fig. 1 MHBC algorithm flow

\subsection{Simulation results and analysis}

This paper designs two simulation experiments, one is to verify the optimization performance of the proposed MHBC algorithm for multi-objective problems, and the other one is to verify the effectiveness of the MHBC algorithm to solve the problem of HAVS formation. 
Simulation Experiment 1 There are UAVs with multiple resource capabilities, see table 1. Existing 4 target groups and the task resource requirements see table 2. The MHBC algorithm proposed in this paper has the local search performance of $\mathrm{ABC}$ algorithm and the global search performance of SA algorithm. MHBC algorithm and NSGA- II algorithm were used to solve the model respectively, and one solution is randomly selected from the Pareto solution set obtained from the two methods, the schemes are shown in table 3.

Table 1. Resources ability of MAV/UAV

\begin{tabular}{|l|c|}
\hline & Resource capability vector \\
\hline$h_{1}$ & 6.56 .62 .53 .42 .75 .75 .63 .4 \\
$h_{2}$ & 6.55 .22 .42 .71 .56 .66 .84 .9 \\
$h_{3}$ & 6.65 .23 .75 .74 .53 .44 .72 .8 \\
$h_{4}$ & 1.55 .53 .42 .76 .55 .83 .61 .1 \\
$h_{5}$ & 8.32 .24 .84 .22 .14 .31 .54 .7 \\
$h_{6}$ & 6.33 .64 .62 .52 .84 .53 .64 .9 \\
$h_{7}$ & 5.83 .34 .13 .52 .74 .42 .54 .9 \\
$h_{8}$ & 5.52 .22 .36 .66 .64 .72 .72 .7 \\
$h_{9}$ & 8.23 .52 .62 .71 .84 .53 .43 .6 \\
$h_{10}$ & 2.23 .55 .52 .46 .24 .42 .25 .7 \\
$h_{11}$ & 1.24 .32 .43 .54 .75 .82 .14 .6 \\
$h_{12}$ & 2.24 .65 .54 .56 .55 .12 .84 .6 \\
$h_{13}$ & 8.13 .12 .55 .66 .73 .72 .81 .9 \\
$h_{14}$ & 2.25 .64 .65 .96 .43 .11 .54 .6 \\
$h_{15}$ & 1.42 .42 .63 .55 .63 .12 .84 .3 \\
$h_{16}$ & 5.12 .72 .83 .65 .64 .52 .64 .8 \\
$h_{17}$ & 2.15 .33 .13 .51 .82 .64 .25 .2 \\
$h_{18}$ & 3.94 .32 .12 .53 .81 .62 .14 .3 \\
$h_{19}$ & 5.13 .52 .64 .32 .85 .93 .63 .2 \\
$h_{20}$ & 6.43 .55 .54 .66 .15 .56 .12 .6 \\
\hline
\end{tabular}

Table 2. Resources demand of alliance tasks

\begin{tabular}{|c|c|c|}
\hline TASK & & Resource requirements of task \\
\hline \multirow{6}{*}{$T_{1}$} & $t_{1}$ & \multirow{6}{*}{$\begin{array}{rllllllll}2.4 & 2.1 & 3.6 & 2.6 & 1.6 & 6.4 & 1.6 & 3.0 \\
3.6 & 2.6 & 4.5 & 2.4 & 0.2 & 4.2 & 0.2 & 3.4 \\
0.1 & 2.2 & 0.5 & 0.6 & 0.3 & 0.5 & 5.8 & 0.6 \\
0.2 & 2.1 & 0.2 & 0.3 & 0.3 & 0.1 & 5.2 & 0.26 .6 \\
0.5 & 2.1 & 2.3 & 0.1 & 0.3 & 0.5 & 3.6 & 0.4 \\
0.3 & 0.3 & 0.1 & 5.5 & 2.4 & 2.3 & 0.3 & 0.0\end{array}$} \\
\hline & $t_{2}$ & \\
\hline & $t_{3}$ & \\
\hline & $t_{4}$ & \\
\hline & $t_{5}$ & \\
\hline & $t_{6}$ & \\
\hline \multirow{7}{*}{$T_{2}$} & $t_{7}$ & $\begin{array}{llllllll}5.1 & 2.1 & 0.2 & 0.3 & 0.3 & 0.4 & 2.5 & 0.3\end{array}$ \\
\hline & $t_{8}$ & 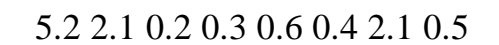 \\
\hline & $t_{9}$ & $\begin{array}{llllllll}0.5 & 0.6 & 0.2 & 0.5 & 0.8 & 2.4 & 2.6 & 0.5\end{array}$ \\
\hline & $t_{10}$ & $\begin{array}{llllllll}0.1 & 0.5 & 0.2 & 0.5 & 0.5 & 1.4 & 0.1 & 0.2\end{array}$ \\
\hline & $t_{11}$ & $\begin{array}{llllllll}0.5 & 0.1 & 0.2 & 0.5 & 0.3 & 3.7 & 0.4 & 2.8\end{array}$ \\
\hline & $t_{12}$ & 0.20 .10 .30 .60 .82 .40 .12 .2 \\
\hline & $t_{13}$ & $\begin{array}{llllllll}0.1 & 0.2 & 0.3 & 4.4 & 2.1 & 2.5 & 0.5 & 0.6\end{array}$ \\
\hline \multirow{5}{*}{$T_{3}$} & $t_{14}$ & $\begin{array}{llllllll}4.2 & 0.1 & 5.3 & 0.2 & 0.1 & 2.2 & 0.1 & 2.5\end{array}$ \\
\hline & $t_{15}$ & $\begin{array}{llllllll}0.1 & 3.6 & 0.2 & 0.5 & 0.4 & 0.1 & 2.2 & 0.3\end{array}$ \\
\hline & $t_{16}$ & $\begin{array}{llllllll}0.1 & 1.5 & 2.1 & 0.2 & 0.3 & 0.5 & 3.6 & 0.1\end{array}$ \\
\hline & $t_{17}$ & 0.10 .30 .62 .62 .13 .50 .50 .6 \\
\hline & $t_{18}$ & $\begin{array}{llllllll}0.2 & 0.1 & 0.3 & 0.3 & 0.1 & 1.5 & 0.5 & 4.3\end{array}$ \\
\hline \multirow{6}{*}{$T_{4}$} & $t_{19}$ & \multirow{6}{*}{$\begin{array}{llllllllll}0.6 & 4.2 & 0.1 & 0.2 & 0.5 & 0.5 & 2.1 & 0.6 \\
0.1 & 3.2 & 0.1 & 0.2 & 0.3 & 0.1 & 2.2 & 0.1 \\
0.1 & 2.4 & 1.1 & 0.2 & 0.1 & 0.3 & 1.9 & 0.5 \\
0.1 & 0.2 & 0.3 & 2.8 & 1.7 & 1.1 & 0.2 & 0.6 \\
3.9 & 1.5 & 0.8 & 0.1 & 0.3 & 0.1 & 1.4 & 0.2 \\
3.5 & 2.1 & 0.2 & 0.3 & 0.1 & 0.2 & 2.4 & 0.2\end{array}$} \\
\hline & $t_{20}$ & \\
\hline & $t_{21}$ & \\
\hline & $t_{22}$ & \\
\hline & $t_{23}$ & \\
\hline & $t_{24}$ & \\
\hline
\end{tabular}


Table 3. Schemes of alliance formation

\begin{tabular}{|c|c|c|c|c|c|c|c|}
\hline \multicolumn{4}{|c|}{ Scheme Derived from the MHBC } & \multicolumn{4}{c|}{ Scheme Derived from the NSGA- II } \\
\hline alliance & UAV & alliance & UAV & alliance & UAV & alliance & UAV \\
\hline$A_{1}$ & $h_{11} h_{13} h_{15} h_{17} h_{19} h_{20}$ & $A_{2}$ & $h_{3} h_{8} h_{9} h_{16}$ & $A_{1}$ & $h_{2} h_{3} h_{5} h_{14} h_{18}$ & $A_{2}$ & $h_{8} h_{9} h_{10} h_{17}$ \\
$A_{3}$ & $h_{5} h_{10} h_{14} h_{18}$ & $A_{4}$ & $h_{1} h_{2} h_{4}$ & $A_{3}$ & $h_{19} h_{5} h_{4} h_{15} h_{20}$ & $A_{4}$ & $h_{6} h_{11} h_{18}$ \\
\hline \multicolumn{2}{|c|}{$\eta(1147$} & \multicolumn{2}{|c|}{$D_{\eta}: 0.1661$} & \multicolumn{3}{|c|}{$\eta: 1.0413$} & $D_{\eta}: 0.4122$ \\
\hline
\end{tabular}

As can be seen from the schemes, in terms of average resource redundancy index, scheme of MHBC algorithm is slightly larger than Scheme of NSGA- II algorithm. In terms of resource redundancy variance index, scheme obtained by MHBC algorithm is much smaller than the NSGA- II algorithm solution. Since the amount of resources is huge in real warfare, it can be seen that the scheme of MHBC algorithm is better than the scheme of NSGA- algorithm.

Simulation experiment 2 as is known to all, the non-dominated sorting genetic algorithm II (NSGAII [4]) is the main method to solve the multi-objective optimization problem. So the performance of MHBC algorithm is measured compared to NSGA-II algorithm. The evaluation index of multi-objective algorithm performance includes convergence index, coverage index, index of uniformity and algorithm time [5]. Set the upper bound of evolutionary algebra of NSGA- II algorithm and MHBC algorithm to 100 , colony size to 60 . After each algorithm run 30 times independently, the results are shown in figure 4.
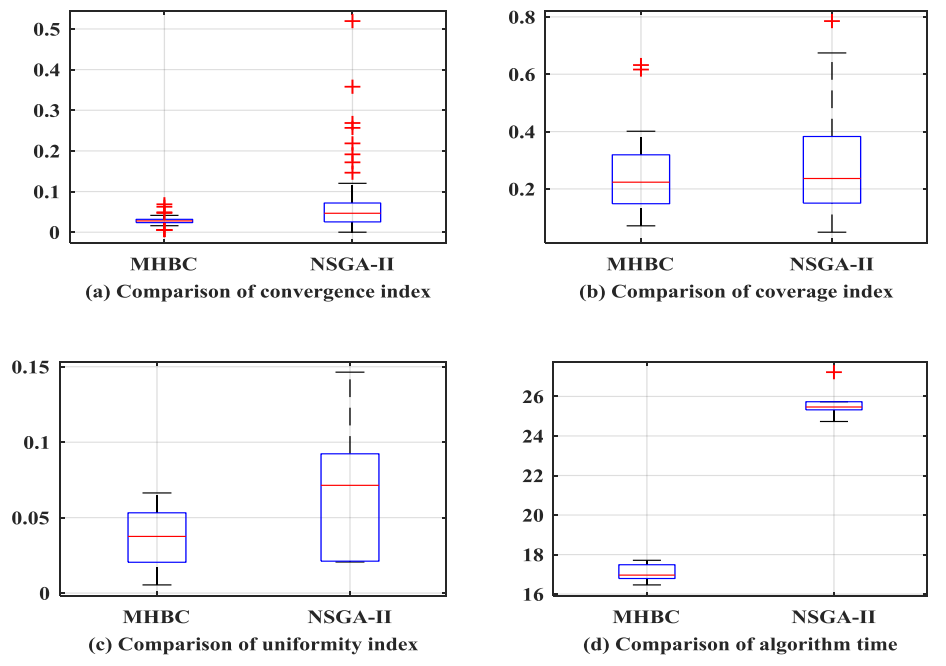

Fig. 2 Comparison of algorithms

As seen in Figure 2, MHBC algorithm is inferior to NSGA- II algorithm in the coverage of solution set, but the convergence, uniformity is better than NSGA- II algorithm, and the computation time is shorter, which is of great significance in actual combat. Therefore, the performance of MHBC algorithm proposed is better the in solving the problem of HAVS formation.

\section{Summary}

This paper explored the problem of HAVS formation, mainly in the following three aspects: (1) The mathematical description method of HAVS is given; (2) The corresponding optimization model is established aim to balance and minimize the resource redundancy of HAVS to generate the best HAVS scheme; (3) MHBC algorithm is proposed and the validity and superiority of the algorithm are proved by experiment. 


\section{References}

[1]. Headquarters. United states air force unmanned aircraft systems flight plan 2009-2047. USAF, Washington DC, 2009.

[2] Karaboga D. An idea based on honey bee swarm for numerical optimization. Technical Report-TR06. Erciyes University, Engineering Faculty, Computer Engineering Department, 2005.

[3] Wang H Y, Wang W B. Hybrid bee colony algorithm with modified search strategy. Systems Engineering and Electronics, Vol.36(2014),No.10, p. 2094-2101.

[4] Deb K, Pratap A, Agarwal S, et al. A fast and elitist multi-objective genetic algorithm: NSGA- II . IEEE Trans. On Evolutionary Computation, Vol.6(2002),No.2, p. 182-197.

[5] Van V D A, Lamont G B. On measuring multi-objective evolutionary algorithm performance// Proc. of the Congress on Evolutionary Computation, Trondheim, Norway, 2009, p, 204-211. 